\title{
EXACT SOLUTIONS AND WRONSKIAN FORMULATION FOR A NEW FORM OF THE $(3+1)$-DIMENSIONAL BKP EQUATION
}

\author{
Li Cheng \\ Normal School, Jinhua Polytechnic \\ Jinhua, 321007, P.R. CHINA
}

\begin{abstract}
The Hirota's bilinear method is used to construct multiple-soliton solutions for a new form of the $(3+1)$-dimensional BKP equation. The resulting solutions involve generic phase shifts and wave frequencies containing some existing choices. By taking the long wave limit approach and extending the real parameters into complex parameters, a few classes of limit solutions and complexitons are generated respectively. Finally, a Wronskian formulation is presented.
\end{abstract}

AMS Subject Classification: 35Q51, 35C10, 37K30

Key Words: the $(3+1)$-dimensional generalized BKP equation, exact solutions, limit solutions

\section{Introduction}

The investigation of the exact solutions of nonlinear evolution equations (NLEEs) plays an important role in the study of nonlinear physical phenomena. Some methods have been developed to obtain the exact solutions, such as the Bäcklund transformation method [1], the Hirota's bilinear method [2], the Painlevé analysis [3] and the Wronskian technique [4, 5]. It is well known that the bilinear method first proposed by Hirota provides us with a comprehensive approach to construct exact solutions [2]. Once a NLEE is written in bilinear form, we are

Received: December 9, 2014

(c) 2015 Academic Publications, Ltd. url: www.acadpubl.eu 
able to derive systematically particular solutions including multiple-soliton solutions. Based on the Hirota bilinear method, a variety of powerful methods have been developed. For example, Ablowitz and Satsuma studied a relationship between soliton and rational solutions of a certain class of NLEEs and developed a method to get rational solutions by taking a long wave limit on soliton solutions obtained by the Hirota bilinear method [6]. Another example, by applying the simplified Hirota bilinear method and extending the real parameters into complex parameters, Wazwaz et al. have obtained nonsingular complexiton solutions for two higher-dimensional nonlinear equations [7]. Recently, the multiple exp-function method [8] has been proposed as a generalization of Hirota's perturbation scheme [2]. Ma et al. have applied the multiple exp-function algorithm to construct multiple wave solutions to the $(3+1)$-dimensional generalized KP and BKP equation [9]. Additionally, according to the Hirota bilinear form, the Wronskian technique is an efficient method to establish multi-soliton solutions for NLEEs in Wronskian form [10, 11].

The aim of this paper is to study a new form of the $(3+1)$-dimensional BKP equation presented by

$$
u_{x x x y}+\alpha\left(u_{x} u_{y}\right)_{x}+\left(u_{x}+u_{y}+u_{z}\right)_{t}-\left(u_{x x}+u_{y y}+u_{z z}\right)=0
$$

where $\alpha$ is a non-zero parameter. First Wazwaz [12] has applied the simplified Hereman-Nuseir form to obtain one and two soliton solutions for Eq. (1.1), Second he has develop specific constraints that guarantee the existence of multiple soliton solutions for Eq. (1.1). In this paper, we would like to use the Hirota's bilinear method, the long wave limit approach and the Wronskian technique to shed light on diversity of exact solutions to Eq. (1.1).

The framework of this paper is as follows. In Section 2, we apply the Hirota's bilinear method to construct multiple soliton solutions to Eq. (1.1). In addition, by taking the long wave limit approach and extending the real parameters into complex parameters, rational solutions, complexitons, positons and negatons are generated. In Section 3, based on the Wronskian formulation of the KdV equation [13], a broad set of sufficient conditions consisting of systems of linear partial differential equations is presented which guarantees that the Wronskian determinant solves Eq. (1.1) in the bilinear form. Our conclusion and remarks are given in Section 4 . 


\section{Soliton and Limit Solutions}

Under the dependent variable transformation

$$
u=\frac{6}{\alpha}(\ln f)_{x}
$$

equation (1.1) is mapped into a Hirota bilinear equation

$$
\left(D_{x}^{3} D_{y}+D_{y} D_{t}+D_{x} D_{t}+D_{z} D_{t}-D_{x}^{2}-D_{y}^{2}-D_{z}^{2}\right) f \cdot f=0,
$$

where $D_{x}, D_{y}, D_{z}$ and $D_{t}$ are Hirota bilinear differential operators [2]. Equivalently, we have

$$
\begin{gathered}
\left(f_{x x x y}+f_{y t}+f_{x t}+f_{z t}-f_{x x}-f_{y y}-f_{z z}\right) f-f_{x x x} f_{y}-3 f_{x} f_{x x y} \\
+3 f_{x x} f_{x y}-f_{x} f_{t}-f_{y} f_{t}-f_{z} f_{t}+f_{x}^{2}+f_{y}^{2}+f_{z}^{2}=0 .
\end{gathered}
$$

\subsection{One-Soliton and Rational Solutions}

Following the Hirota's bilinear method, the one-soliton solutions of Eq. (1.1) read

$$
u=\frac{6}{\alpha}(\ln f)_{x}=\frac{6 k_{1} e^{k_{1} x+l_{1} y+m_{1} z-w_{1} t+\xi_{1}^{0}}}{\alpha\left(1+e^{\left.k_{1} x+l_{1} y+m_{1} z-w_{1} t+\xi_{1}^{0}\right)}\right.},
$$

with

$$
f_{1}=1+e^{\xi_{1}},
$$

where $\xi_{1}=k_{1} x+l_{1} y+m_{1} z-w_{1} t+\xi_{1}^{0}, k_{1}, l_{1}, m_{1}$ are constants, and the dispersion relation being satisfied

$$
w_{1}=\frac{k_{1}^{3} l_{1}-k_{1}^{2}-l_{1}^{2}-m_{1}^{2}}{k_{1}+l_{1}+m_{1}} .
$$

The fact that one can recover rational solutions relies on our freedom of choosing the arbitrary phase constants $\xi_{1}^{0}$. For example, in (2.4), if we set $e^{\xi_{1}^{0}}=-1, l_{1}=\gamma_{1} k_{1}, m_{1}=\gamma_{2} k_{1}$, and take the limit of $k_{1} \rightarrow 0$, then we find

$$
f_{1}=-k_{1} \theta_{1}+o\left(k_{1}^{2}\right),
$$

where $\theta_{1}=x+\gamma_{1} y+\gamma_{2} z+\frac{1+\gamma_{1}^{2}+\gamma_{2}^{2}}{1+\gamma_{1}+\gamma_{2}} t$. Since $u$ is given (2.1), $f_{1}$ is equivalent to $\theta_{1}$. Thus, the first class of rational solutions to Eq. (1.1) is

$$
u=\frac{6}{\alpha \theta_{1}} .
$$




\subsection{Two-Soliton, Rational and Complexiton Solutions}

To determine the two-soliton solutions, we follow and use the auxiliary function

$$
f_{2}=1+e^{\xi_{1}}+e^{\xi_{2}}+a_{12} e^{\xi_{1}+\xi_{2}}
$$

where

$$
\xi_{i}=k_{i} x+l_{i} y+m_{i} z-w_{i} t+\xi_{i}^{0}, i=1,2 .
$$

Applying Hirota's perturbation scheme leads to

$$
a_{12}=-\frac{b_{12}}{c_{12}}
$$

where

$$
\begin{aligned}
b_{12}= & \left(k_{1}-k_{2}\right)^{3}\left(l_{1}-l_{2}\right)+\left(l_{1}-l_{2}\right)\left(w_{2}-w_{1}\right)+\left(k_{1}-k_{2}\right)\left(w_{2}-w_{1}\right) \\
& +\left(m_{1}-m_{2}\right)\left(w_{2}-w_{1}\right)-\left(k_{1}-k_{2}\right)^{2}-\left(l_{1}-l_{2}\right)^{2}-\left(m_{1}-m_{2}\right)^{2}, \\
c_{12}= & \left(k_{1}+k_{2}\right)^{3}\left(l_{1}+l_{2}\right)+\left(l_{1}+l_{2}\right)\left(-w_{2}-w_{1}\right)+\left(k_{1}+k_{2}\right)\left(-w_{2}-w_{1}\right) \\
& +\left(m_{1}+m_{2}\right)\left(-w_{2}-w_{1}\right)-\left(k_{1}+k_{2}\right)^{2}-\left(l_{1}+l_{2}\right)^{2}-\left(m_{1}+m_{2}\right)^{2},
\end{aligned}
$$

and

$$
w_{i}=\frac{k_{i}^{3} l_{i}-k_{i}^{2}-l_{i}^{2}-m_{i}^{2}}{k_{i}+l_{i}+m_{i}} .
$$

It is easy to find that the phase shift $a_{12}$ depends on all coefficients $k_{i}, l_{i}$ and $m_{i}, i=1,2$, of the spatial variables $x, y$ and $z$, respectively. This means that two-soliton solutions are determined for $k_{i}, l_{i}$ and $m_{i}$ being free parameters. We will explore some interesting reductions of this kind of phase shifts later when discussing rational and complexiton solutions.

Firstly, if we take the choices

$$
l_{i}=\beta_{1} k_{i}, m_{i}=\beta_{2} k_{i}, i=1,2,
$$

where $\beta_{1}$ and $\beta_{2}$ are constants. This yields the wave frequencies

$$
w_{i}=\frac{\beta_{1} k_{i}^{3}-\left(1+\beta_{1}^{2}+\beta_{2}^{2}\right) k_{i}}{1+\beta_{1}+\beta_{2}}, i=1,2,
$$

and the phase shift

$$
a_{12}=\frac{\left(k_{1}-k_{2}\right)^{2}}{\left(k_{1}+k_{2}\right)^{2}}
$$


In the case of (2.11), a special reduction

$$
k_{i}=l_{i}=m_{i}, i=1,2,
$$

presents the choice for the two-soliton solutions discussed in Ref. [12].

The long wave limit approach can be applied to $f_{2}$. Under the choices (2.11), choosing $e^{\xi_{1}^{0}}=-e^{\xi_{2}^{0}}=\frac{k_{1}+k_{2}}{k_{1}-k_{2}}$ and taking the limit $k_{i} \rightarrow 0(i=1,2)$ in (2.7), we get

$$
f_{2}=-\frac{1}{6} k_{1} k_{2}\left(k_{1}+k_{2}\right) \theta_{2}+o\left(k^{4}\right)
$$

where

$$
\theta_{2}=\frac{12 \beta_{1}}{1+\beta_{1}+\beta_{2}} t+\left(x+\beta_{1} y+\beta_{2} z+\frac{1+\beta_{1}^{2}+\beta_{2}^{2}}{1+\beta_{1}+\beta_{2}} t\right)^{3} .
$$

The constant $-\frac{1}{6} k_{1} k_{2}\left(k_{1}+k_{2}\right)$ can be neglected in (2.14) as before. Thus, the second class of rational solutions is

$$
u=\frac{18}{\alpha \theta_{2}}\left(x+\beta_{1} y+\beta_{2} z+\frac{1+\beta_{1}^{2}+\beta_{2}^{2}}{1+\beta_{1}+\beta_{2}} t\right)^{2} .
$$

By virtue of the two-soliton solutions with the phase shift given in (2.13), we next discuss and illustrate complexiton and positon solutions of Eq. (1.1).

Case 1 Assuming

$$
k_{1}=a+\mathrm{i} b, k_{2}=a-\mathrm{i} b,(a, b \in R),
$$

and noticing expressions $(2.8),(2.11),(2.12)$ and (2.13), we have

$$
\xi_{1}=\Omega_{1}+\mathrm{i} \psi_{1}+\xi_{1}^{0}, \quad \xi_{2}=\Omega_{1}-\mathrm{i} \psi_{1}+\xi_{2}^{0}, \quad a_{12}=-\frac{b^{2}}{a^{2}},
$$

where

$$
\begin{aligned}
& \Omega_{1}=a x+\beta_{1} a y+\beta_{2} a z-\frac{\beta_{1}\left(a^{3}-3 a b^{2}\right) t-\left(1+\beta_{1}{ }^{2}+\beta_{2}{ }^{2}\right) a t}{1+\beta_{1}+\beta_{2}}, \\
& \psi_{1}=b x+\beta_{1} b y+\beta_{2} b z+\frac{\beta_{1}\left(b^{3}-3 a^{2} b\right) t+\left(1+\beta_{1}{ }^{2}+\beta_{2}{ }^{2}\right) b t}{1+\beta_{1}+\beta_{2}} .
\end{aligned}
$$

Substituting (2.17) into (2.7) and using the choice $e^{\xi_{1}^{0}}=e^{\xi_{2}^{0}}=\frac{a}{b}$, we can now compute

$$
f_{2}=1+\frac{2 a}{b} e^{\Omega_{1}} \cos \psi_{1}-e^{2 \Omega_{1}} .
$$


Consequently, we obtain a class of explicit solution of Eq. (1.1) as follows:

$$
u=\frac{12\left(a^{2} e^{\Omega_{1}} \cos \psi_{1}-a b e^{\Omega_{1}} \sin \psi_{1}-a b e^{2 \Omega_{1}}\right)}{\alpha\left(b+2 a e^{\Omega_{1}} \cos \psi_{1}-b e^{2 \Omega_{1}}\right)},
$$

the parameters $a$ and $b$ are suitably chosen.

When $a b \neq 0$, expression (2.19) is a class of complexiton solutions of Eq. (1.1), i.e., solution involving two kinds of transcendental functions - exponential functions and trigonometric functions.

Furthermore, in (2.18), $f_{2}$ is also written as

$$
f_{2}=\frac{2 a}{b}\left(e^{\Omega_{1}} \cos \psi_{1}+\frac{b}{2 a}\left(1-e^{2 \Omega_{1}}\right)\right),
$$

where the multiplicative factor $\frac{2 a}{b}$ as a constant does not affect the solution (2.1) and can be neglected. Setting the limit $a \rightarrow 0$ and employing the transformation (2.1), a class of limit solutions of Eq. (1.1) is given by

$$
u=\frac{6}{\alpha}\left(\ln \left(\cos \widehat{\psi}_{1}-b \widehat{\Omega}_{1}\right)\right)_{x}=\frac{-6 b\left(\sin \widehat{\psi}_{1}+1\right)}{\alpha\left(\cos \widehat{\psi}_{1}-b \widehat{\Omega}_{1}\right)},
$$

where

$$
\begin{aligned}
& \widehat{\Omega}_{1}=x+\beta_{1} y+\beta_{2} z+\frac{3 \beta_{1} b^{2} t+\left(1+\beta_{1}^{2}+\beta_{2}^{2}\right) t}{1+\beta_{1}+\beta_{2}}, \\
& \widehat{\psi}_{1}=b x+\beta_{1} b y+\beta_{2} b z+\frac{\beta_{1} b^{3} t+\left(1+\beta_{1}{ }^{2}+\beta_{2}^{2}\right) b t}{1+\beta_{1}+\beta_{2}} .
\end{aligned}
$$

We easily see that (2.21) involves only one kind of transcendental functionstrigonometric functions, and so it gives a class of positon solutions.

Case 2 The same limiting procedure can be applied to the case

$$
k_{1}=a+\mathrm{i} b, k_{2}=-a+\mathrm{i} b,(a, b \in R) .
$$

Substituting (2.22) into (2.13) and taking $e^{\xi_{1}^{0}}=\frac{a \mathrm{i}}{b}, e^{\xi_{2}^{0}}=-\frac{a \mathrm{i}}{b}$ in (2.7), we find

$$
f_{2}=\frac{2 b \mathrm{i}}{a}\left(e^{\mathrm{i} \psi_{1}} \sinh \Omega_{1}+\frac{a}{2 b \mathrm{i}}\left(1-e^{2 \mathrm{i} \psi_{1}}\right)\right)
$$

where $\Omega_{1}, \psi_{1}$ are defined by (2.17). Taking the limit $b \rightarrow 0$ and using the transformation (2.1), the resulting negaton solutions read

$$
u=\frac{6}{\alpha}\left(\ln \left(\sinh \widetilde{\Omega}_{1}-a \widetilde{\psi}_{1}\right)\right)_{x}=\frac{6 a\left(\cosh \widetilde{\Omega}_{1}-1\right)}{\alpha\left(\sinh \widetilde{\Omega}_{1}-a \widetilde{\psi}_{1}\right)} .
$$


where

$$
\begin{aligned}
& \widetilde{\Omega}_{1}=a x+\beta_{1} a y+\beta_{2} a z-\frac{\beta_{1} a^{3} t-\left(1+\beta_{1}{ }^{2}+\beta_{2}{ }^{2}\right) a t}{1+\beta_{1}+\beta_{2}}, \\
& \widetilde{\psi}_{1}=x+\beta_{1} y+\beta_{2} z-\frac{3 \beta_{1} a^{2} t-\left(1+\beta_{1}{ }^{2}+\beta_{2}{ }^{2}\right) t}{1+\beta_{1}+\beta_{2}} .
\end{aligned}
$$

The graphs of complexiton and positon solutions with specific values being chosen for the parameters are presented in Figure 1, which show some singularities of the solutions.

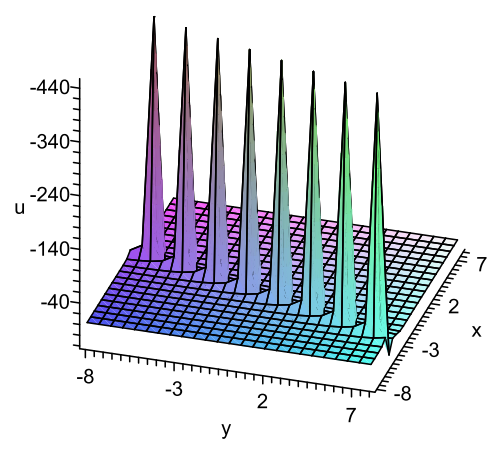

(a)

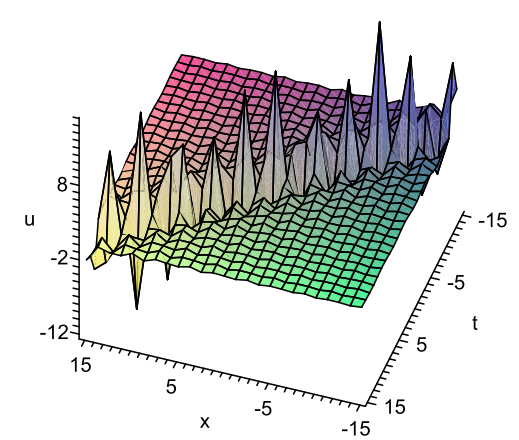

(b)

Figure 1: (a) The plot of the complexiton solution given by (2.19) with parameters: $a=2, b=1, \beta_{1}=\frac{1}{3}, \beta_{2}=1, \alpha=1, z=0, t=2$. (b) The plot of positon solution given by (2.21) with parameters: $b=-2, \beta_{1}=-\frac{1}{3}, \beta_{2}=1, \alpha=2, z=0, y=2$.

Secondly, let us take the choices

$$
l_{1}=\beta_{1} k_{1}, l_{2}=-\beta_{1} k_{2}, m_{1}=\beta_{2} k_{1}, m_{2}=\delta k_{2},
$$

where $\beta_{1}, \beta_{2}$ and $\delta$ are constants. This leads to the wave frequencies

$$
w_{1}=\frac{\beta_{1} k_{1}^{3}-\left(1+\beta_{1}^{2}+\beta_{2}^{2}\right) k_{1}}{1+\beta_{1}+\beta_{2}}, w_{2}=\frac{-\beta_{1} k_{2}^{3}-\left(1+\beta_{1}^{2}+\delta^{2}\right) k_{2}}{1-\beta_{1}+\delta},
$$

and the phase shift $a_{12}=1$.

Furthermore, choosing $e^{\xi_{1}^{0}}=e^{\xi_{2}^{0}}=-1$ and taking the limit $k_{i} \rightarrow 0(i=1,2)$ in (2.7), we have

$$
f_{2}=\theta_{3} \theta_{4}
$$


where

$$
\begin{aligned}
& \theta_{3}=\left(x+\beta_{1} y+\beta_{2} z+\frac{\left(1+\beta_{1}^{2}+\beta_{2}^{2}\right) t}{1+\beta_{1}+\beta_{2}}\right), \\
& \theta_{4}=\left(x-\beta_{1} y+\delta z+\frac{\left(1+\beta_{1}^{2}+\delta^{2}\right) t}{1-\beta_{1}+\delta}\right) .
\end{aligned}
$$

Then the third class of rational solutions is determined by

$$
u=\frac{6}{\alpha \theta_{3} \theta_{4}}\left(2 x+\left(\beta_{2}+\delta\right) z+\frac{\left(1+\beta_{1}^{2}+\beta_{2}^{2}\right) t}{1+\beta_{1}+\beta_{2}}+\frac{\left(1+\beta_{1}^{2}+\delta^{2}\right) t}{1-\beta_{1}+\delta}\right) .
$$

\subsection{Three-Soliton and Rational Solutions}

We now consider three-soliton solutions

$$
u=\frac{6}{\alpha}\left(\ln f_{3}\right)_{x}
$$

with $f_{3}$ being defined by

$$
\begin{gathered}
f_{3}=1+e^{\xi_{1}}+e^{\xi_{2}}+e^{\xi_{3}}+a_{12} e^{\xi_{1}+\xi_{2}}+a_{23} e^{\xi_{2}+\xi_{3}}+a_{13} e^{\xi_{1}+\xi_{3}}+a_{123} e^{\xi_{1}+\xi_{2}+\xi_{3}} \\
a_{123}=a_{12} a_{13} a_{23}
\end{gathered}
$$

where

$$
\xi_{i}=k_{i} x+l_{i} y+m_{i} z-w_{i} t+\xi_{i}^{0}, 1 \leq i \leq 3 .
$$

We would like to search for three-soliton solutions with the selection of

$$
w_{i}=\frac{k_{i}^{3} l_{i}-k_{i}^{2}-l_{i}^{2}-m_{i}^{2}}{k_{i}+l_{i}+m_{i}}, 1 \leq i \leq 3
$$

and

$$
a_{i j}=-\frac{b_{i j}}{c_{i j}}, 1 \leq i<j \leq 3
$$

where

$$
\begin{aligned}
b_{i j}= & \left(k_{i}-k_{j}\right)^{3}\left(l_{i}-l_{j}\right)+\left(l_{i}-l_{j}\right)\left(w_{j}-w_{i}\right)+\left(k_{i}-k_{j}\right)\left(w_{j}-w_{i}\right) \\
& +\left(m_{i}-m_{j}\right)\left(w_{j}-w_{i}\right)-\left(k_{i}-k_{j}\right)^{2}-\left(l_{i}-l_{j}\right)^{2}-\left(m_{i}-m_{j}\right)^{2}, \\
c_{i j}= & \left(k_{i}+k_{j}\right)^{3}\left(l_{i}+l_{j}\right)+\left(l_{i}+l_{j}\right)\left(-w_{j}-w_{i}\right)+\left(k_{i}+k_{j}\right)\left(-w_{j}-w_{i}\right) \\
& +\left(m_{i}+m_{j}\right)\left(-w_{j}-w_{i}\right)-\left(k_{i}+k_{j}\right)^{2}-\left(l_{i}+l_{j}\right)^{2}-\left(m_{i}+m_{j}\right)^{2} .
\end{aligned}
$$


Since the Eq. (1.1) is not completely integrable [12], we need to determine conditions to generated three-soliton solutions.

The first class of three-soliton solutions is associated with the choices

$$
l_{i}=\beta_{1} k_{i}, m_{i}=\beta_{2} k_{i}, 1 \leq i \leq 3,
$$

where $\beta_{1}$ and $\beta_{2}$ are constants. This yields the wave frequencies

$$
w_{i}=\frac{\beta_{1} k_{i}^{3}-\left(1+\beta_{1}{ }^{2}+\beta_{2}{ }^{2}\right) k_{i}}{1+\beta_{1}+\beta_{2}}, 1 \leq i \leq 3,
$$

and the phase shifts

$$
\begin{aligned}
& a_{i j}=\frac{\left(k_{i}-k_{j}\right)^{2}}{\left(k_{i}+k_{j}\right)^{2}}, 1 \leq i<j \leq 3, \\
& a_{123}=\frac{\left(k_{1}-k_{2}\right)^{2}\left(k_{2}-k_{3}\right)^{2}\left(k_{1}-k_{3}\right)^{2}}{\left(k_{1}+k_{2}\right)^{2}\left(k_{2}+k_{3}\right)^{2}\left(k_{1}+k_{3}\right)^{2}} .
\end{aligned}
$$

Taking

$$
\begin{gathered}
e^{\xi_{1}^{0}}=\frac{\left(k_{1}+k_{2}\right)\left(k_{1}+k_{3}\right)}{\left(k_{1}-k_{2}\right)\left(k_{1}-k_{3}\right)}, e^{\xi_{2}^{0}}=\frac{\left(k_{2}+k_{1}\right)\left(k_{2}+k_{3}\right)}{\left(k_{2}-k_{1}\right)\left(k_{2}-k_{3}\right)}, \\
e^{\xi_{3}^{0}}=\frac{\left(k_{3}+k_{1}\right)\left(k_{3}+k_{2}\right)}{\left(k_{3}-k_{1}\right)\left(k_{3}-k_{2}\right)},
\end{gathered}
$$

and passing to the limit $k_{i} \rightarrow 0(1 \leq i \leq 3)$ in (2.30), we can obtain a class of limit solutions

$$
f_{3}=\theta_{3}^{6}+\frac{60 \beta_{1} t}{1+\beta_{1}+\beta_{2}} \theta_{3}^{3}-720\left(\frac{\beta_{1} t}{1+\beta_{1}+\beta_{2}}\right)^{2},
$$

where $\theta_{3}$ is defined by (2.28). Thus, we also obtain a class of rational solutions of Eq. (1.1) by the transformation (2.1), which is different from the above three types of rational solutions.

The second class of three-soliton solutions is associated with the choices

$$
l_{1}=\beta_{1} k_{1}, l_{2}=-\beta_{1} k_{2}, l_{3}=\beta_{1} k_{3}, m_{1}=\beta_{2} k_{1}, m_{2}=\delta k_{2}, m_{3}=\beta_{2} k_{3},
$$

where $\beta_{1}, \beta_{2}$ and $\delta$ are constants, which leads to the wave frequencies

$$
w_{i}=\frac{\beta_{1} k_{i}^{3}-\left(1+\beta_{1}^{2}+\beta_{2}^{2}\right) k_{i}}{1+\beta_{1}+\beta_{2}}, i=1,3,
$$




$$
w_{2}=\frac{-\beta_{1} k_{2}^{3}-\left(1+\beta_{1}^{2}+\delta^{2}\right) k_{2}}{1-\beta_{1}+\delta},
$$

and the phase shifts

$$
a_{12}=1, a_{13}=\frac{\left(k_{1}-k_{3}\right)^{2}}{\left(k_{1}+k_{3}\right)^{2}}, a_{23}=1
$$

Taking

$$
e^{\xi_{1}^{0}}=\frac{\left(k_{1}+k_{3}\right)}{\left(k_{1}-k_{3}\right)}, e^{\xi_{2}^{0}}=-1, e^{\xi_{3}^{0}}=\frac{\left(k_{3}+k_{1}\right)}{\left(k_{3}-k_{1}\right)},
$$

and passing to the limit $k_{i} \rightarrow 0(1 \leq i \leq 3)$ in $(2.30)$, we have

$$
f_{3}=\left(x-\beta_{1} y+\delta z+\frac{1+\beta_{1}^{2}+\delta^{2}}{1-\beta_{1}+\delta} t\right)\left(\frac{\beta_{1} t}{1+\beta_{1}+\beta_{2}}\right) .
$$

The corresponding class of rational solutions is similiar to the above rational solutions (2.6).

\section{Wronskian Formulation of Eq. (1.1)}

Next, applying the Wronskian technique, we will present the Wronskian formulation to Eq. (1.1). To use this technique, we adopt the compact notation introduced by Freeman and Nimmo [4, 5], they set

$$
\begin{gathered}
W\left(\phi_{1}, \phi_{2}, \cdots, \phi_{N}\right)=\left|\begin{array}{cccc}
\phi_{1} & \phi_{1}^{(1)} & \cdots & \phi_{1}^{(N-1)} \\
\phi_{2} & \phi_{2}^{(1)} & \cdots & \phi_{2}^{(N-1)} \\
\vdots & \vdots & \vdots & \vdots \\
\phi_{N} & \phi_{N}^{(1)} & \cdots & \phi_{N}^{(N-1)}
\end{array}\right| \\
=|0,1, \cdots, N-1|=|\widehat{N-1}|
\end{gathered}
$$

where $\phi_{i}^{(j)}=\frac{\partial^{j} \phi_{i}}{\partial x^{j}}$

First we state useful results about the $\mathrm{KdV}$ equation

$$
u_{t}-6 u u_{x}+u_{x x x}=0,
$$

which has the bilinear form

$$
\left(D_{x} D_{t}+D_{x}^{4}\right) f \cdot f=0,
$$


through the transformation $u=-2(\ln f)_{x x}$. Equivalently, we have

$$
f f_{x t}-f_{x} f_{t}+f f_{x x x x}-4 f_{x} f_{x x x}+3 f_{x x}^{2}=0 .
$$

In Ref. [13], the following Theorem 1 has been proved:

Theorem 1. Assume that a group of functions $\phi_{i}=\phi_{i}(x, t),(1 \leq i \leq N)$ satisfies the two sets of conditions

$$
\begin{array}{r}
-\phi_{i, x x}=\sum_{j=1}^{N} \lambda_{i j}(t) \phi_{j}, 1 \leq i \leq N, \\
\phi_{i, t}=-4 \phi_{i, x x x}+\zeta(t) \phi_{i}, 1 \leq i \leq N,
\end{array}
$$

simultaneously, where $\lambda_{i j}(t)$ are arbitrary differentiable real functions of $t$ and $\zeta(t)$ is an arbitrary continuous real function of $t$. Then $f=|\widehat{N-1}|$ defined by (3.1) solves the bilinear KdV equation (3.3).

Using the Theorem 1, we would like to present a broad set of sufficient conditions which make the Wronskian determinant a solution to the bilinear BKP equation (2.2).

Theorem 2. Assume that a group of functions $\phi_{i}=\phi_{i}(x, y, z, t),(1 \leq i \leq N)$ satisfies the following conditions:

$$
\begin{aligned}
& \phi_{i, x x}=\sum_{j=1}^{N} \lambda_{i j}(t) \phi_{j}, \\
& \phi_{i, y}=\beta_{1} \phi_{i, x}, \\
& \phi_{i, z}=\beta_{2} \phi_{i, x}, \\
& \phi_{i, t}=\beta_{3} \phi_{i, x}+\beta_{4} \phi_{i, x x x}+\zeta(t) \phi_{i},
\end{aligned}
$$

with

$$
\beta_{3}=\frac{1+\beta_{1}^{2}+\beta_{2}^{2}}{1+\beta_{1}+\beta_{2}}, \beta_{4}=\frac{-4 \beta_{1}}{1+\beta_{1}+\beta_{2}},
$$

where $\beta_{1}, \beta_{2}$ are two real constants not to be zero, $1+\beta_{1}+\beta_{2} \neq 0, \lambda_{i j}(t)$ are arbitrary differentiable real functions of $t$, and $\zeta(t)$ is an arbitrary continuous real function of $t$. Then the Wronskian determinant $f=|\widehat{N-1}|$ defined by (3.1) solves the bilinear Eq.(2.2). 
Proof. By using the conditions (3.6), we can now compute that

$$
\begin{aligned}
f_{y} & =\beta_{1} f_{x}, f_{x y}=\beta_{1} f_{x x}, f_{x x y}=\beta_{1} f_{x x x}, f_{x x x y}=\beta_{1} f_{x x x x}, f_{y y}=\beta_{1}^{2} f_{x x}, \\
f_{z} & =\beta_{2} f_{x}, f_{z z}=\beta_{2}^{2} f_{x x}, f_{t}=\beta_{3} f_{x}+\beta_{4} f_{(\widetilde{3 x})}+N \zeta f \\
f_{x t} & =\beta_{3} f_{x x}+\beta_{4} f_{(\widetilde{3 x}) x}+N \zeta f_{x}, f_{y t}=\beta_{1} \beta_{3} f_{x x}+\beta_{1} \beta_{4} f_{(\widetilde{3 x}) x}+\beta_{1} N \zeta f_{x}, \\
f_{z t} & =\beta_{2} \beta_{3} f_{x x}+\beta_{2} \beta_{4} f_{(\widetilde{3 x}) x}+\beta_{2} N \zeta f_{x}
\end{aligned}
$$

where

$$
\begin{gathered}
f_{(\widetilde{3 x})}=|\widehat{N-4}, N-2, N-1, N|-|\widehat{N-3}, N-1, N+1|+|\widehat{N-2}, N+2|, \\
f_{(\widetilde{3 x}) x}=|\widehat{N-5}, N-3, N-2, N-1, N|-|\widehat{N-3}, N, N+1|+|\widehat{N-2}, N+3| .
\end{gathered}
$$

Substituting these derivatives into (2.3) and using the condition (3.6e), we have

$$
\begin{aligned}
&\left(f_{x x x y}+f_{y t}+f_{x t}+f_{z t}-f_{x x}-f_{y y}-f_{z z}\right) f-f_{x x x} f_{y}-3 f_{x} f_{x x y} \\
&+3 f_{x x} f_{x y}-f_{x} f_{t}-f_{y} f_{t}-f_{z} f_{t}+f_{x}^{2}+f_{y}^{2}+f_{z}^{2} \\
&=\beta_{1}\left(-4 f f_{\widetilde{3 x}) x}+4 f_{x} f_{(\widetilde{3 x})}+f f_{x x x x}-4 f_{x} f_{x x x}+3 f_{x x}^{2}\right) .
\end{aligned}
$$

By virtue of Theorem 1, expression (3.7) is equal to zero. Therefore, we have shown that $f=|\widehat{N-1}|$ solves the bilinear Eq.(2.2).

\section{Conclusion and Remarks}

In summary, by using the Hirota's bilinear method and the long wave limit approach, we have presented multi-soliton, rational, positon, negaton and complexiton solutions. Although we need to make additional constraint for the parameters to guarantee the existence of multi-soliton solutions, the newly presented generic phase shifts and wave frequencies in this paper lead to the richness and diversity of exact solutions for the considered Eq. (1.1).

On the other hand, based on the Wronskian formulation of the KdV equation, we have established a Wronskian formulation for Eq. (1.1), with all generating functions for matrix entries satisfying a linear system of partial differential equations involving free parameters. Of course, the obtained Wronskian solution formulas of Eq. (1.1) allow us to construct rational solutions. However, our rational solutions obtained by taking the long wave limit approach contain the rational solutions generated from Wronskian formulation. 


\section{Acknowledgments}

This work is supported by the scientific research project of Zhejiang education department (No. Y201224998).

\section{References}

[1] W.X. Ma and W. Strampp, Bilinear forms and Bäcklund transformations of the perturbation systems, Phys. Lett. A, 341 (2005), 441-449.

[2] R. Hirota, The Direct Method in Soliton Theory, Cambridge University Press, Cambridge, (2004).

[3] Y. Zhang, Y. Song, L. Cheng, J.Y. Ge and W.W. Wei, Exact solutions and Painlevé analysis of a new (2+1)-dimensional generalized KdV equation, Nonlinear Dyn. 68 (2012), 445-458.

[4] N.C. Freeman and J.J.C. Nimmo, Soliton solutions of the Korteweg-de Vries and Kadomtsev- Petviashvili equations: the Wronskian technique, Phys. Lett. A 95 (1983), 1-3.

[5] J.J.C. Nimmo and N.C. Freeman, A method of obtaining the N-soliton solution of the Boussinesq equation in terms of a Wronskian, Phys. Lett. A 95 (1983), 4-6.

[6] M.J. Ablowitz and J. Satsuma, Solitons and rational solutions of nonlinear evolution equations, J. Math. Phys. 19 (1978) 2180-2186.

[7] A.M. Wazwaz and Q.L. Zha, Nonsingular complexiton solutions for two higher-dimensional fifth-order nonlinear integrable equation. Phys. Scr. $\mathbf{8 8}$ (2013), 025001.

[8] W.X. Ma, T.W. Huang and Y. Zhang, A multiple exp-function method for nonlinear differential equations and its application, Phys. Scr. 82 (2010), 065003.

[9] W.X. Ma and Z.N. Zhu, Solving the $(3+1)$-dimensional generalized KP and BKP equations by the exp-function algorithm. Appl. Math. Comput. 218 (2012), 11871-11879.

[10] W.X. Ma, A. Abdeljabbar and M.G. Asaad, Wronskian and Grammian solutions to a (3+1)- dimensional generalized KP equation, Appl. Math. Comput. 217 (2011), 10016-10023. 
[11] K. Cui, New Wronskian Form of the $N$-Soliton Solution to a $(2+1)$ Dimensional Breaking Soliton Equation, Chin. Phys. Lett. 29 (2012), 060508.

[12] A.M. Wazwaz, Two forms of $(3+1)$-dimensional B-type KadomtsevPetviashvili equation: multiple soliton solutions. Phys. Scr. 86 (2012), 035007.

[13] W.X. Ma and Y.C. You, Solving the Korteweg-de Vries equation by its bilinear form: Wronskian solutions, Trans. Amer. Math. Soc. 357 (2005), 1753-1778. 